\title{
Investigation on Harmonic Tuning for Active Ku-Band Rectangular Dielectric Resonator Antennas
}

\author{
Anda Guraliuc, ${ }^{1}$ Giuliano Manara, ${ }^{1}$ Paolo Nepa, ${ }^{1}$ Giuseppe Pelosi, ${ }^{2}$ and Stefano Selleri ${ }^{2}$ \\ ${ }^{1}$ Department of Information Engineering, University of Pisa, Via G. Caruso 16, 56126 Pisa, Italy \\ ${ }^{2}$ Department of Electronics and Telecommunications, University of Florence, Via C. Lombroso 6/17, 50137 Firenze, Italy
}

Correspondence should be addressed to Stefano Selleri, stefano.selleri@unifi.it

Received 21 September 2007; Revised 7 March 2008; Accepted 16 April 2008

Recommended by Hisashi Morishita

A slot-coupled rectangular dielectric resonator antenna (DRA) operating in the $14-14.5 \mathrm{GHz}$ frequency band is investigated as a possible radiating element for an active integrated antenna of a transmitting phased array. The effectiveness of the resonator shape factor on achieving harmonic tuning is addressed. Simulation results show that the DRA shape factor can be used to provide a fine tuning of the DRA input impedance both at the fundamental frequency and its first harmonics, so synthesizing the proper load for the optimization of the microwave amplifier power-added efficiency (PAE).

Copyright (c) 2008 Anda Guraliuc et al. This is an open access article distributed under the Creative Commons Attribution License, which permits unrestricted use, distribution, and reproduction in any medium, provided the original work is properly cited.

\section{INTRODUCTION}

During the latest years, an increasing research interest has been devoted to dielectric resonator antennas (DRAs) [1-3] in the microwave and millimeter frequency ranges, since they show several attractive features over conventional antennas, such as small size, high radiation efficiency, light weight, intrinsic simple structure, high power-handling capability, extreme flexibility in terms of resonator shapes and feeding techniques. Moreover, different radiation patterns can be achieved with the same resonator by exciting different radiating modes, and for a given resonant frequency, they can realize miniaturized antennas if a dielectric resonator with high permittivity is used. Also, when compared with microstrip or slot antennas exhibiting a comparable layout complexity, the DRA has a relatively larger impedance bandwidth and does not suffer of conductor and surface wave losses. DRA development is also strictly related to the recent technological advances on low-loss dielectric materials exhibiting a wide range of dielectric permittivity (up to $\varepsilon_{r}=100$ ). As far as phased arrays are concerned, the compactness of DRAs (obtained by high-permittivity dielectric resonators) allows smaller interelement separation compared with many other antenna types, and it results in reduced scan losses.

Although DRAs have been deeply investigated, previous studies have been mainly focused on radiated field char- acteristics, radiation efficiency, and impedance bandwidth widening. Conversely, reduced attention has been devoted to the investigation of DRA arrays [4-7] and harmonic tuning of active DRAs [8]. On the other hand, the tuning of the antenna input impedance at the fundamental frequency as well as at the first harmonics is strongly recommended in transmitting active integrated antennas (AIAs) $[9,10]$, where high power added efficiency (PAE) values are required. Moreover, the harmonics generated by the power amplifier will be radiated by the antenna if the latter does not exhibit a sufficiently high reflection coefficient at those frequencies. As a consequence, harmonic radiation causes system efficiency reduction and electromagnetic interference with other radio systems. Therefore, an efficient design of an AIA that does not consider an impedance matching network between the antenna and the active device must require the tuning of the antenna input impedance at the first and second harmonic, and, possibly, at the third harmonic too, even if latter requirement is less restrictive since third harmonics power levels are anyway quite low. Several configurations have been devised for harmonic tuning of microstrip antennas, which are based on the use of holes, pins, or slots, as well as on the shaping of the radiating patch $[11,12]$.

In this paper, harmonic tuning of a rectangular DRA for an active-phased array antenna working in the 14$14.5 \mathrm{GHz}$ frequency band (VSWR $<-10 \mathrm{~dB}$ ) is addressed. It is shown that the input impedance control at the harmonic 


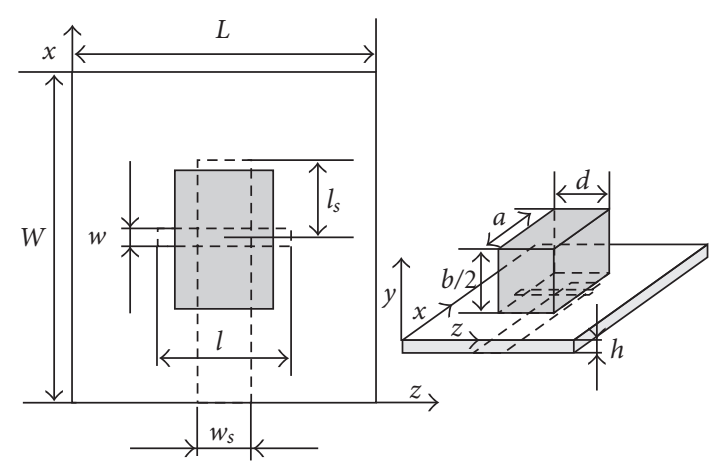

FIGURE 1: Geometry of the slot-coupled rectangular DRA, comprehensive of finite ground plane and feeding microstrip.

frequencies can be effectively carried out through a modification of the rectangular resonator shape factor. A DRAphased array represents an effective alternative to printed technology in $\mathrm{Ku}$-band satellite broadband communication systems, mainly due to their compactness, high efficiency, compatibility with microwave integrated circuits, and low scan losses. A slot coupling feeding technique has been chosen, since it is the most suitable for large DRA arrays, where a relatively simple circuit integration is required, as compared with other feeding mechanisms. Moreover, since a ground plane isolates the feeding structure from the radiator, the radiation characteristics are mainly those of the dielectric resonator and surface waves are not excited.

The paper is organized as follow. In Section 1, a brief overview on rectangular DRAs is reported to summarize their main features. Then in Section 2 it is shown that a fine tuning of the input impedance of a slot-coupled rectangular DRA at the first harmonics can be easily obtained by changing the resonator length-to-width and length-toheight ratios. Indeed, it is possible to vary the above ratios of the DRA to obtain a wide variability range for the input impedance at the second harmonic, without seriously affecting the input impedance and radiation patterns at the center frequency $(14.25 \mathrm{GHz})$. Simulation results have been obtained by using the Ansoft HFSS software package, which has been demonstrated being an accurate tool for the analysis of rectangular slot-coupled DRAs.

\section{A BRIEF OVERVIEW ON RECTANGULAR DIELECTRIC RESONATOR ANTENNAS}

The basic configuration of a rectangular $a \times d \times b / 2$ DRA over a finite conducting ground plane is shown in Figure 1. Theoretical and experimental investigations on various shapes of DRAs have been reported in the open literature [1$3]$, showing that the rectangular DRA has some advantages over the cylindrical and hemispherical DRAs [13]. Indeed, by properly choosing the dielectric resonator sizes, mode degeneracy can be avoided and cross-polarization can be taken under control. Moreover, compared to the above DRA shapes, rectangular DRAs are easier to fabricate.

The DRA impedance bandwidth can be increased (lower radiation $Q$ factor) by using low-permittivity dielectric res- onators or by resorting to parasitic elements, at the cost of increasing the DRA size and fabrication complexity, respectively [1-3]. Several nonhomogeneous DRA configurations have also been proposed such as stacked, notched, or multisegment configurations [1-3] as well as rectangular resonators with a metal coating [14]. In addition, for a given resonant frequency, in a rectangular DRA the two aspect ratios (length-to-height and length-to-width) can be chosen independently, and then used to achieve impedance bandwidth widening [15].

Different feeding mechanisms have been theoretically and experimentally investigated [1], as, for example, a coaxial probe, a microstrip feedline, a coplanar waveguide, a rectangular waveguide, which make the DRAs amenable to integration with different existing electronic technologies. Recently, a differential feed has also been proposed [16], to avoid using a balun when connecting the antenna to a differential circuit for the common mode rejection. As far as circular polarization (CP) DRAs are concerned, single-point feed systems have been suggested, such as quasisquared and crossed DRAs, which exhibit a CP bandwidth up to 7\% [2].

Finally, although most of the work has been done for single element optimization, the advantages of using linear or planar DRA arrays to implement phased arrays with beam steering capabilities have also been investigated. In planar arrays, the compactness of DRAs using high-permittivity dielectric resonators allows for a small separation between the array elements, which is often required in electronically beam steering antenna or when a proper excitation tapering must be implemented. In [17], it has been shown that when used as an active antenna, a rectangular DRA shows superior tolerance levels with respect to fabrication and larger impedance bandwidth, as compared to an active microstrip patch antenna. However, in [17] impedance matching networks comprising an open-circuit stub have been used, and the harmonic tuning issue is not faced with. In [8], harmonic tuning of a rectangular DRA has been achieved by adding a pair of parasitic slots adjacent to the coupling slot located between the microstrip line and the dielectric resonator.

\section{SHAPE-FACTOR-BASED HARMONIC TUNING}

The effectiveness of the shape factor on achieving the harmonic tuning of a rectangular DRA is shown in this section, where the design of a slot-coupled rectangular DRA suitable for a Ku-band phased array is used as a test case. The rectangular $a \times d \times b / 2$ DRA fed through a rectangular slot coupled with a microstrip line is shown in Figure 1. The resonator relative permittivity has been set to $\varepsilon_{r}=20$. The DRA is placed above a ground plane, and is centered with respect to the $l \times w$ nonresonant rectangular slot. The antenna is fed through a microstrip line $(25 \Omega$ characteristic impedance, as requested by the chosen PA layout for maximum PAE) of width $w_{s}=1.6 \mathrm{~mm}$, which is printed on the bottom of a grounded substrate (thickness and relative permittivity equal to $h=0.5 \mathrm{~mm}$ and $\varepsilon_{r}=$ 10.2 , resp.) whose sizes are $L=17 \mathrm{~mm}, W=14 \mathrm{~mm}$. The antenna has to operate in the $\mathrm{TE}_{111}^{z}$ mode at a frequency 


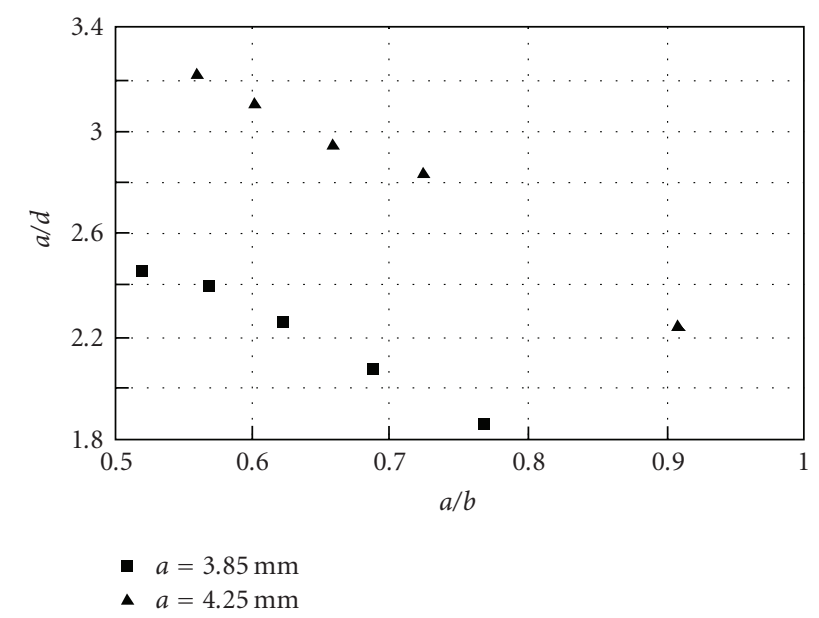

(a)

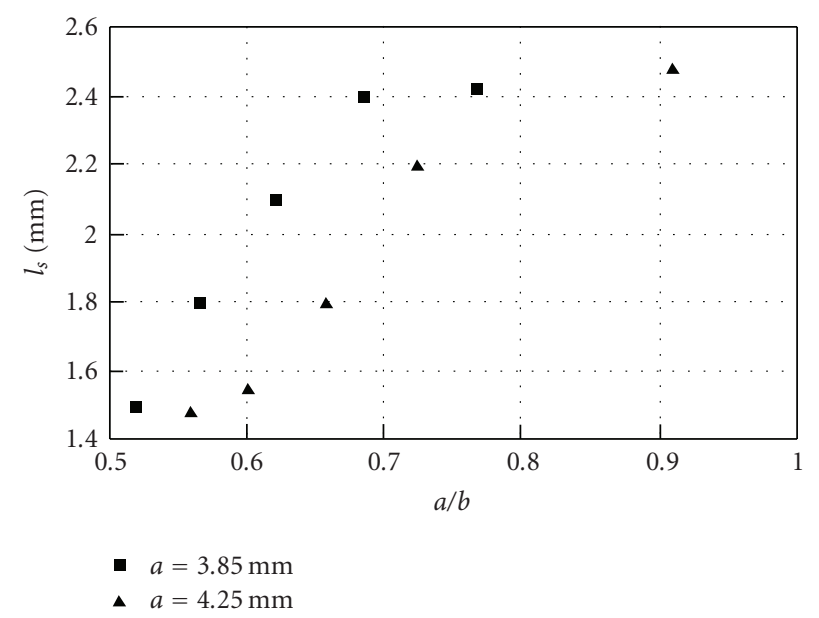

(b)

FIGURE 2: Length-to-width ratio (a) and open-circuit stub length (b) versus length-to-height ratio for a rectangular DRA operating in the fundamental $\mathrm{TE}_{111}^{z}$ mode at $f_{0}=14.25 \mathrm{GHz}$.

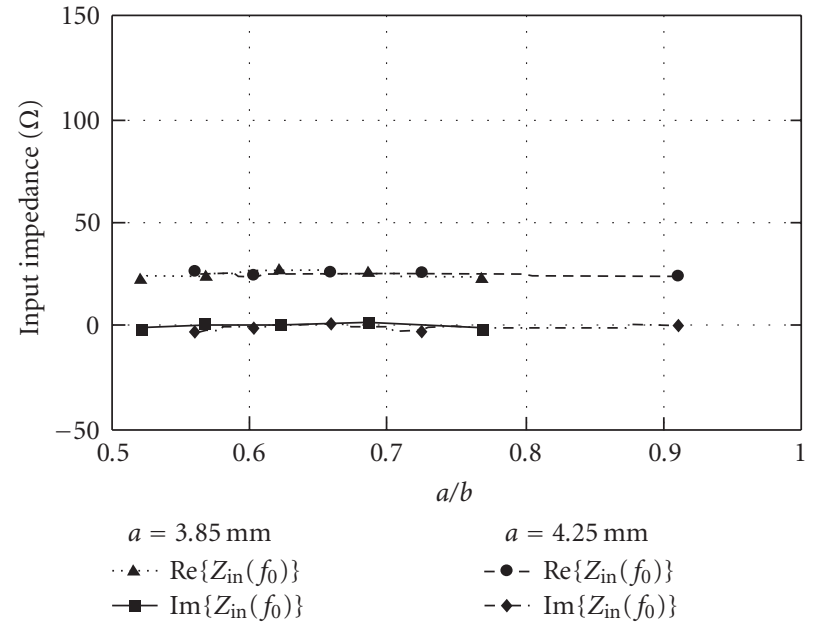

FIGURE 3: Real and imaginary values of the DRA input impedance at the fundamental frequency versus length-to-height ratio, for two values of the resonator length: $a=3.85 \mathrm{~mm}$ and $a=4.25 \mathrm{~mm}$.

$f_{0}=14.25 \mathrm{GHz}$, with a VSWR $<-10 \mathrm{~dB}$ in a $500 \mathrm{MHz}$ frequency band (3.5\% percentage bandwidth).

As far as the fundamental "magnetic dipole" mode of a rectangular DRA is concerned, efficient analytical formulas have been derived to estimate the resonator dimensions for a given resonant frequency [13], which exhibit a percentage error less than a few percent. Specifically, for a given $(a, b)$ pair, it is possible to analytically estimate the value of $d$ corresponding to a resonant frequency close to $f_{0}=$ 14.25 GHz. A sample of numerical results is shown in Figure 2(a), where the length-to-width ratio $a / d$ versus the length-to-height ratio $a / b$ is shown for two different values of the DRA length: $a=3.85 \mathrm{~mm}$ and $a=4.25 \mathrm{~mm}$. Tables 1 and 2 show the numerical values of these setups. By means of HFSS simulations, it has been verified that all the geometrical configurations shown in Figure 2 provide the
TABLe 1: First set of DRA dimensions $(a, b, d)$ considered for a rectangular DRA operating in the fundamental $\mathrm{TE}_{111}^{z}$ mode at $f_{0}=$ $14.25 \mathrm{GHz}$.

\begin{tabular}{lccc}
\hline ID & $a[\mathrm{~mm}]$ & $b[\mathrm{~mm}]$ & $d[\mathrm{~mm}]$ \\
\hline I.1 & & 5.02 & 2.06 \\
I.2 & & 5.62 & 1.85 \\
I.3 & 3.85 & 6.20 & 1.70 \\
I.4 & & 6.80 & 1.60 \\
I.5 & & 7.42 & 1.56 \\
\hline
\end{tabular}

TABLe 2: Second set of DRA dimensions $(a, b, d)$ considered for a rectangular DRA operating in the fundamental $\mathrm{TE}_{111}^{z}$ mode at $f_{0}=$ $14.25 \mathrm{GHz}$.

\begin{tabular}{lccc}
\hline ID & $a[\mathrm{~mm}]$ & $b[\mathrm{~mm}]$ & $d[\mathrm{~mm}]$ \\
\hline II.1 & & 4.68 & 1.90 \\
II.2 & & 5.88 & 1.54 \\
II.3 & 4.25 & 6.48 & 1.44 \\
II.4 & 7.08 & 1.37 \\
II.5 & & 7.68 & 1.32 \\
\hline
\end{tabular}

correct resonant frequency, when the DRA is fed through a slot with $l=2.2 \mathrm{~mm}$ and $w=0.3 \mathrm{~mm}$. A fine tuning of the length of the open-circuit stub at the end of the feeding line, $l_{s}$, was adopted to optimize the return loss performance at $f_{0}=14.25 \mathrm{GHz}$ (see Figure 2(b)).

The values of the input impedance at the fundamental frequency, $f_{0}=14.25 \mathrm{GHz}$, are shown in Figure 3 for all the aspect ratios analyzed in Figure 2. As apparent, a pretty good impedance matching at $25 \Omega$ has been achieved for all the configurations listed in Tables 1 and 2. The input impedance values obtained at $2 f_{0}=28.5 \mathrm{GHz}$ and $3 f_{0}=42.75 \mathrm{GHz}$ are also shown in Figures 4(a) and 4(b), respectively. As apparent, by varying the DRA-shape factor, a wide range of imaginary part values can be provided, leading to either 


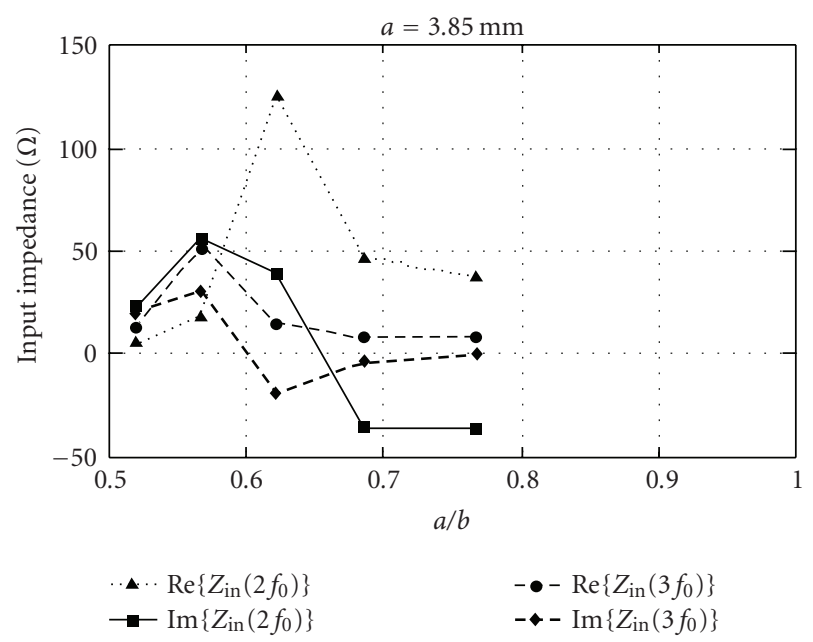

(a)

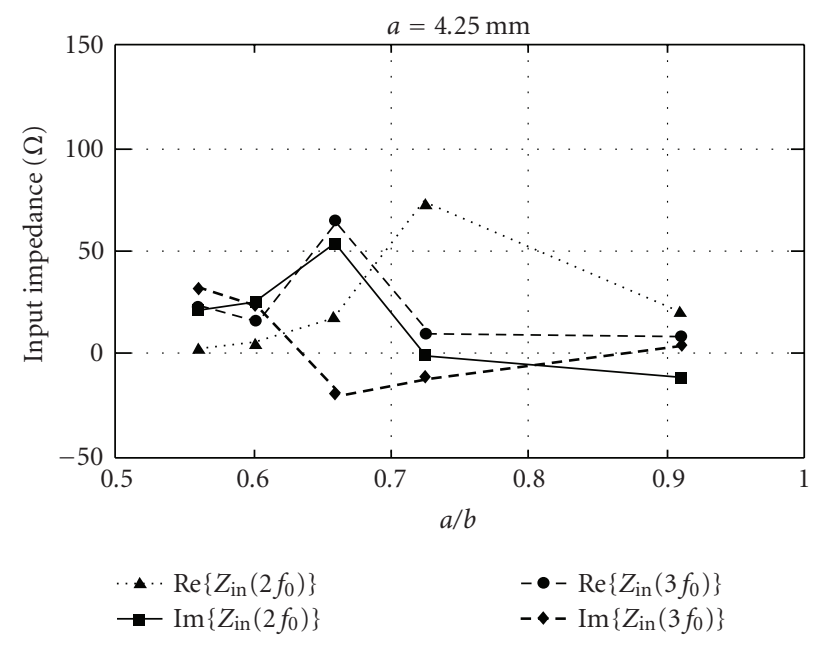

(b)

FIGURE 4: Real and imaginary values of the DRA input impedance at the second and third harmonic frequencies versus length-to-height ratio, for two values of the resonator length: (a) $a=3.85 \mathrm{~mm}$ and (b) $a=4.25 \mathrm{~mm}$.
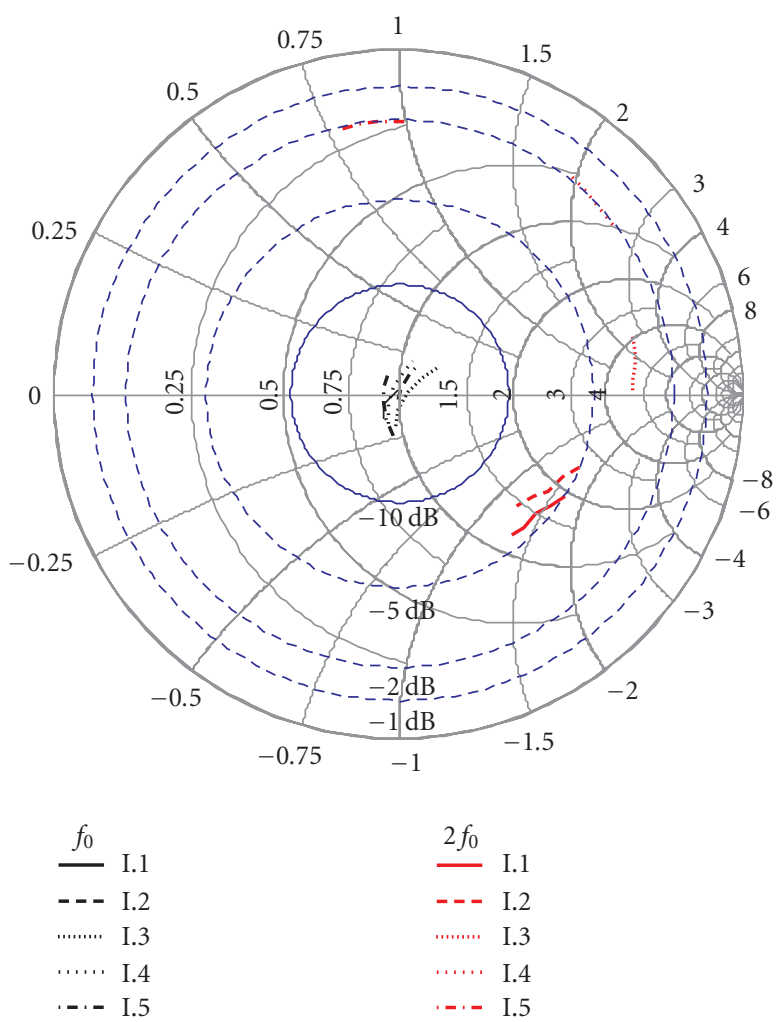

FIGURE 5: Input impedance over a $200 \mathrm{MHz}$ frequency band around $f_{0}$ and $2 f_{0}$ for the DRA with $a=3.85 \mathrm{~mm}$ (the geometrical configurations I.1 to I.5 are those listed in Table 1).

inductive or capacitive input reactance. In Figure 5, the input impedance values are plotted on a Smith Chart over a $200 \mathrm{MHz}$ frequency range around $f_{0}$ and $2 f_{0}$, for the $a=$ $3.85 \mathrm{~mm}$ (case I). For frequencies around $f_{0}$, the curves are clustered around the Smith Chart origin, so demonstrating that a quite good impedance matching condition is always met independently of the specific DRA configuration chosen between those in Table 1 . For frequencies around $2 f_{0}$, the return loss is always greater than $-4 \mathrm{~dB}$ (except for case I.1). It is also apparent that by changing the DRA configuration (between the different aspect ratios in Figure 2) it is possible to control the phase of the return loss, namely, the reactive component of the DRA input impedance. The latter is an important feature in an AIA design since the antenna must not only reject higher harmonics, but also exhibit a proper reflection coefficient phase to optimize the PAE. Similar considerations apply to the results in Figure 6, where the input impedance values over a $200 \mathrm{MHz}$ frequency range around $f_{0}$ and $2 f_{0}$ are plotted on a Smith Chart for the $a=$ $4.25 \mathrm{~mm}$ (case II). In this case, it is apparent how a couple of DRA configurations (cases II.1 and II.2) do not guarantee a return loss higher than $-2 \mathrm{~dB}$ at the second harmonic $2 f_{0}$.

In Figure 6, the percentage impedance bandwidth (VSWR $<-10 \mathrm{~dB}$ ) for the configurations in Figure 2 is shown, to demonstrate that beside controlling the input impedance at the frequencies $2 f_{0}$ and $3 f_{0}$, we can also met the bandwidth specifications (percentage bandwidth greater than 3.5\%). It is worth noting that the percentage bandwidth decreases at the increasing of the length-to-height ratio, as already found in [15] where the degree of freedom represented by the DRA-shape factor was adopted to enlarge the antenna impedance bandwidth.

Finally, Figure 8 shows the radiation patterns in the principal planes (both copolar and cross-polar components), for the DRA geometrical configurations, when $a=4.25 \mathrm{~mm}$ and for two different values of the length-to-height ratio: the II. 1 configuration, which has $a / b=(a / b)_{\max }=0.91$, and the II.5 configuration, which has $a / b=(a / b)_{\min }=$ 0.55 . It is apparent that the radiation patterns do not vary significantly with respect to the length-to-height ratio $a / b$, as expected, since the same mode is excited (it is worth noting that the slot-fed DRA radiates as a short magnetic dipole 

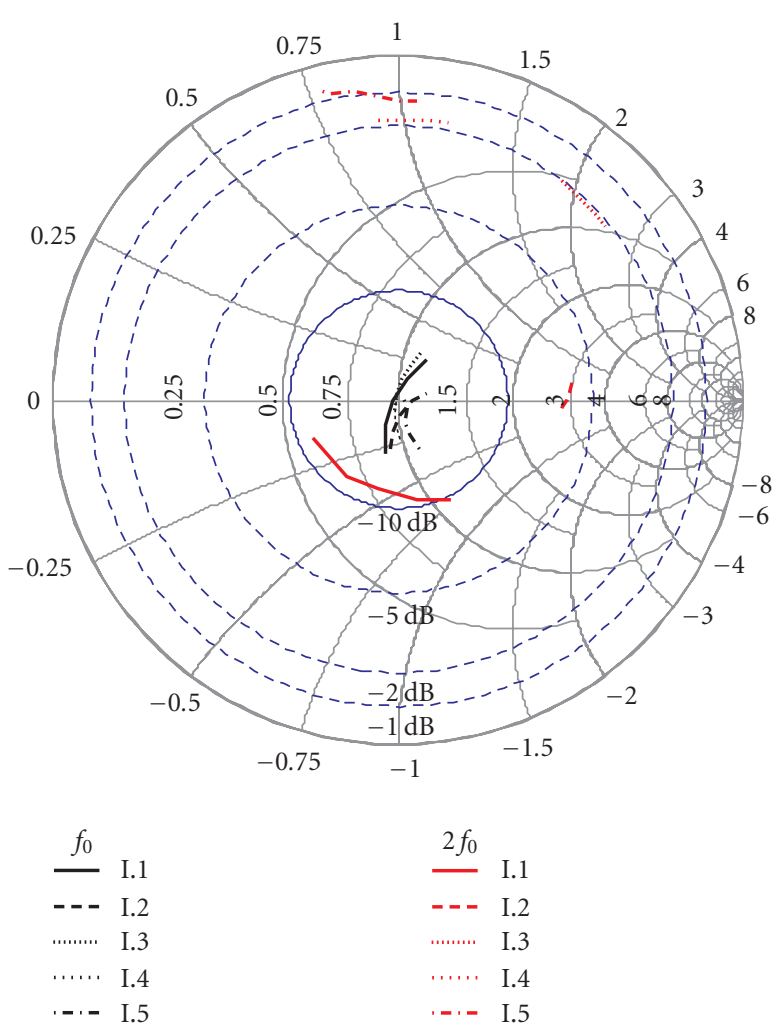

FIgURE 6: Input impedance over a $500 \mathrm{MHz}$ frequency band around $f_{0}$ and $2 f_{0}$ for the DRA with $a=4.25 \mathrm{~mm}$ (the geometrical configurations II.1 to II.5 are those listed in Table 2).

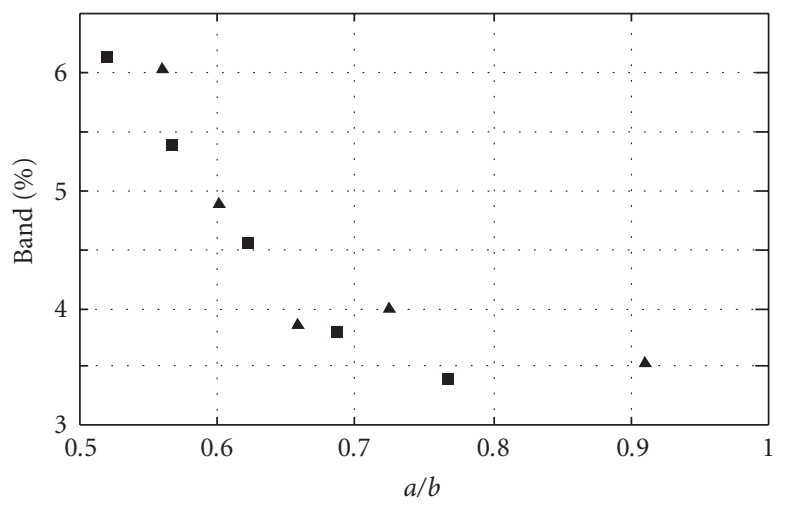

- $a=3.85 \mathrm{~mm}$

A $a=4.25 \mathrm{~mm}$

FIgURE 7: Percentage bandwidth (VSWR $<-10 \mathrm{~dB}$ ) versus the length-to-height ratio (the other geometrical parameters are those shown in Figure 2).

parallel to the slot itself, in its lowest order mode). The crosspolar components are mainly due to the field scattered at the borders of the finite ground plane. A small asymmetry in the E-plane is due to the presence of the microstrip line feeding the slot and to radiating surface waves excited at the open circuit stub. The larger cross-polar level is around $15 \mathrm{~dB}$ and $20 \mathrm{~dB}$ in the $E$-plane and $H$-plane, respectively, and it is

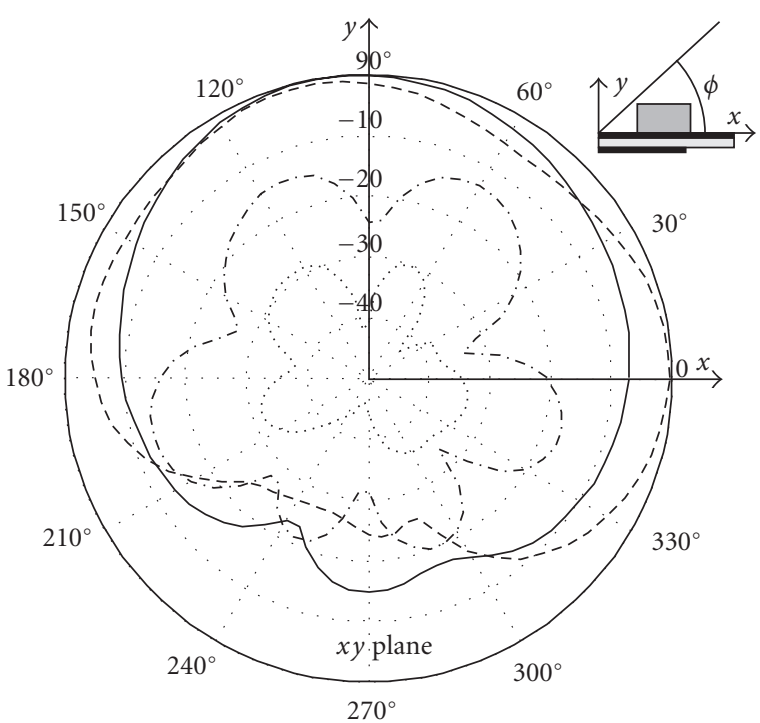

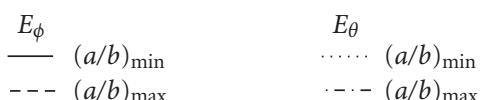

(a)

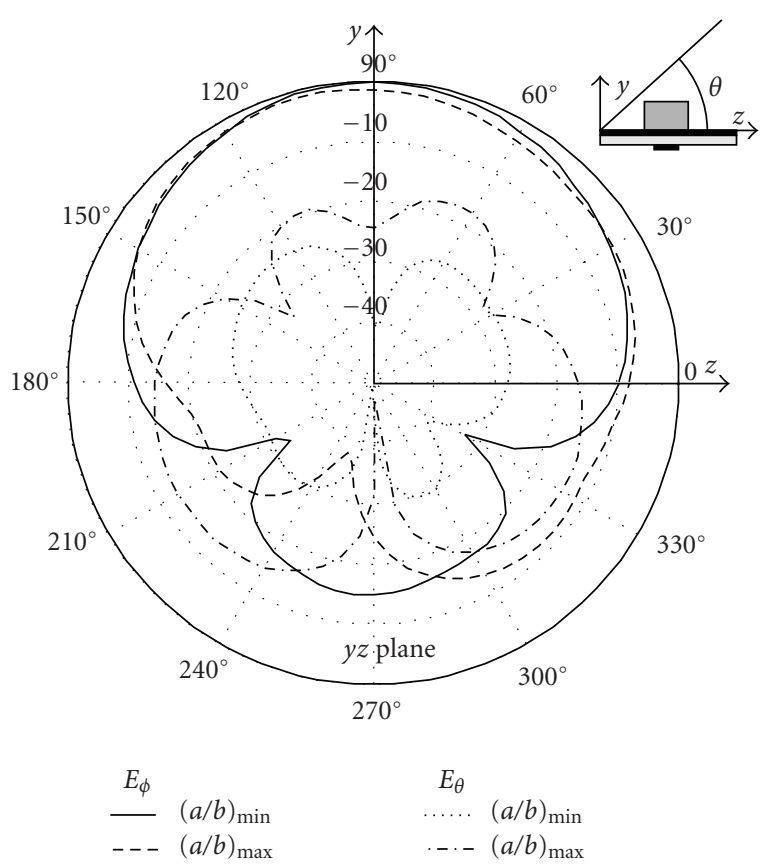

(b)

FIGURE 8: Radiation patterns for a DRA with $a=4.25 \mathrm{~mm}$, and $a / b=\min (a / b)=0.55$ or $a / b=\max (a / b)=0.91$ : (a) $x y$ plane (E-plane); (b) $y z$ plane ( $H$-plane).

associated with configuration II.1 exhibiting the maximum value of the length-to-height ratio, that is, $a / b=(a / b)_{\max }=$ 0.91 . This result is in agreement with [6], where it is stated that higher cross-polar levels are obtained with lower values of the length-to-width ratio, $a / d$ (from Tables 1 and 2 it results that $a / d=3.21$ if $a / b=(a / b)_{\min }=0.55$, while $a / d=2.23$ if $\left.a / b=(a / b)_{\max }=0.91\right)$. 


\section{CONCLUSIONS}

It has been shown that the aspect ratios of a $\mathrm{Ku}$-band slot-coupled rectangular DRA can be used to control the antenna input impedance at the fundamental and first harmonic frequencies, without any significant change in antenna radiation patterns. Besides, the three dimensions of the dielectric resonator, only a fine tuning of the stub length is needed to optimize the return loss at the fundamental frequency, while the slot geometrical parameters can remain unchanged. Work is in progress to estimate the effect of a poor mechanical contact between the DRA and the ground plane (air gap), which can significantly modify the DRA input impedance especially at the higher harmonics as well as the effect of misaglinments in centering the DRA over the slot.

\section{ACKNOWLEDGMENT}

This work was partially supported by the Italian Ministry of Education, University and Research, under the PRIN research project no. 005098437 (Active Integrated Antennas for High-Efficiency Mobile Terminals).

\section{REFERENCES}

[1] K. M. Luk and K. W. Leung, Dielectric Resonator Antennas, Research Studies Press, Oxford, UK, 2003.

[2] A. Petosa, A. Ittipiboon, Y. M. M. Antar, D. Roscoe, and M. Cuhaci, "Recent advances in dielectric-resonator antenna technology," IEEE Antennas and Propagation Magazine, vol. 40, no. 3, pp. 35-47, 1998.

[3] Y. M. M. Antar and D. Guha, "Wireless communication antennas: special requirements and new designs of dielectric resonator antennas," in Proceedings of the 23rd Annual Review of Progress in Applied Computational Electromagnetics (ACES '07), Verona, Italy, March 2007.

[4] D. Kajfez and A. A. Kishk, "Dielectric resonator antennapossible candidate for adaptive antenna arrays," in Proceedings of the International Symposium on Telecommunications, Next Generation Networks and Beyond (VITEL '02), Portoroz, Slovenia, May 2002.

[5] A. A. Kishk, "Dielectric resonator antenna elements for array applications," in Proceedings of the IEEE International Symposium on Phased Array Systems and Technology, pp. 300305, Monterey, Calif, USA, October 2003.

[6] A. Petosa, R. Larose, A. Lttipiboon, and M. Cuhaci, "Microstrip-fed array of multisegment dielectric resonator antennas," IEE Proceedings: Microwaves, Antennas and Propagation, vol. 144, no. 6, pp. 472-476, 1997.

[7] N. V. Larsen and O. Breinbjerg, "Analysis of circularly polarized hemispheroidal dielectric resonator antenna phased arrays using the method of auxiliary sources," IEEE Transactions on Antennas and Propagation, vol. 55, no. 8, pp. 2163 2173, 2007.

[8] A. Guraliuc, G. Manara, P. Nepa, G. Pelosi, and S. Selleri, "Harmonic tuning for Ku-band dielectric resonator antennas," IEEE Antenna and Wireless Propagation Letters, vol. 6, pp. 568$571,2007$.

[9] K. Chang, R. A. York, P. S. Hall, and T. Itoh, "Active integrated antennas," IEEE Transactions on Microwave Theory and Techniques, vol. 50, no. 3, pp. 937-944, 2002.
[10] M. J. Cryan, G. R. Buesnel, and P. S. Hall, "Analysis and control of harmonic radiation from active integrated oscillator antennas," IEEE Transactions on Microwave Theory and Techniques, vol. 50, no. 11, pp. 2639-2646, 2002.

[11] H. Kim and Y. J. Yoon, "Microstrip-fed slot antennas with suppressed harmonics," IEEE Transactions on Antennas and Propagation, vol. 53, no. 9, pp. 2809-2817, 2005.

[12] S. Kwon, B. M. Lee, Y. J. Yoon, W. Y. Song, and J.-G. Yook, "A harmonic suppression antenna for an active integrated antenna," IEEE Microwave and Wireless Components Letters, vol. 13, no. 2, pp. 54-56, 2003.

[13] R. K. Mongia and A. Ittipiboon, "Theoretical and experimental investigations on rectangular dielectric resonator antennas," IEEE Transactions on Antennas and Propagation, vol. 45, no. 9, pp. 1348-1356, 1997.

[14] T.-H. Chang and J.-F. Kiang, "Broadband dielectric resonator antenna with metal coating," IEEE Transactions on Antennas and Propagation, vol. 55, no. 5, pp. 1254-1259, 2007.

[15] C. S. De Young and S. A. Long, "Wideband cylindrical and rectangular dielectric resonator antennas," IEEE Antennas and Wireless Propagation Letters, vol. 5, no. 1, pp. 426-429, 2006.

[16] B. Li and K. W. Leung, "On the differentially fed rectangular dielectric resonator antenna," IEEE Transactions on Antennas and Propagation, vol. 56, no. 2, pp. 353-359, 2008.

[17] M. G. Keller, D. J. Roscoe, M. B. Oliver, R. K. Mongia, Y. M. M. Antar, and A. Ittipiboon, "Active aperture-coupled rectangular dielectric resonator antenna," IEEE Microwave and Guided Wave Letters, vol. 5, no. 11, pp. 376-378, 1995. 

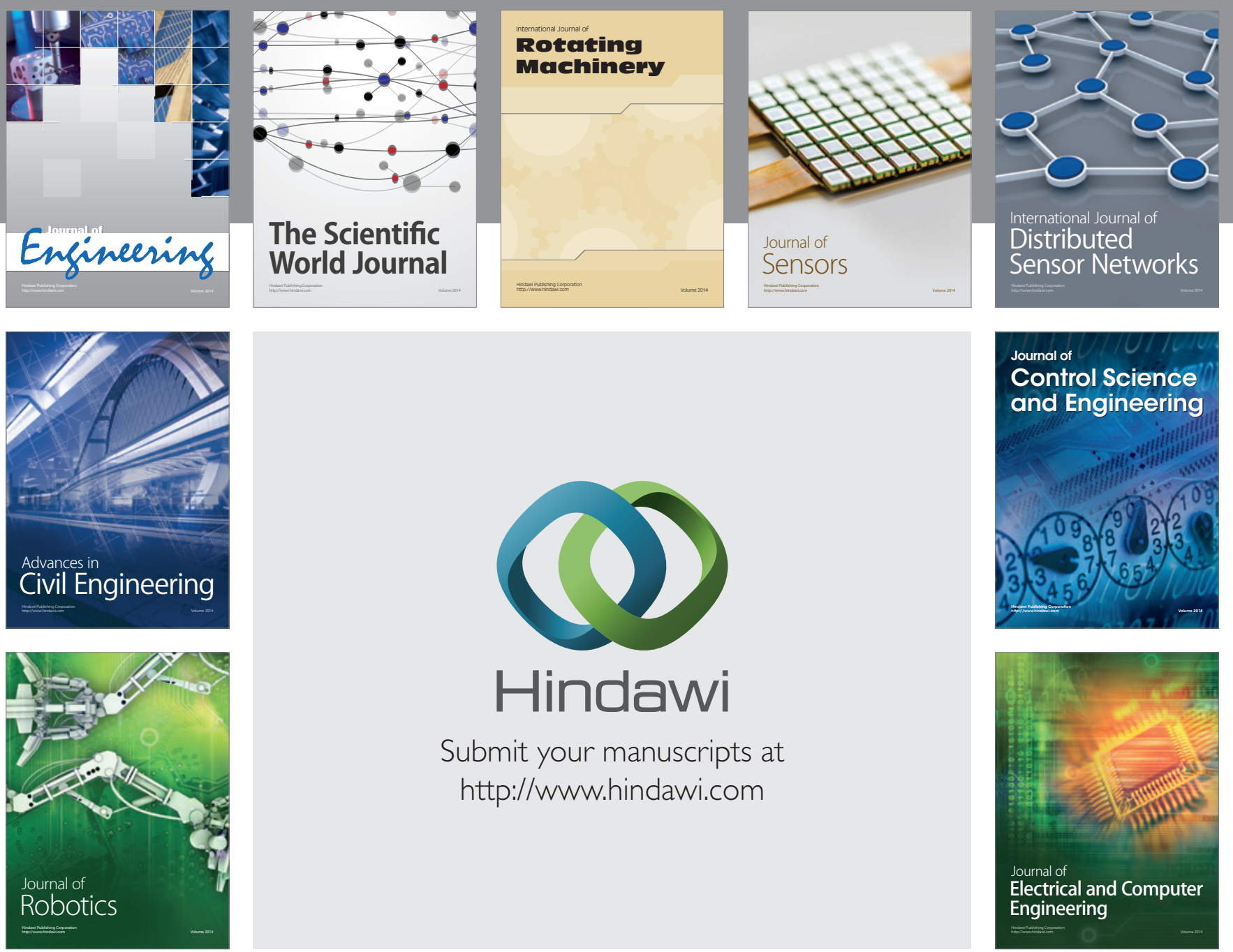

Submit your manuscripts at

http://www.hindawi.com
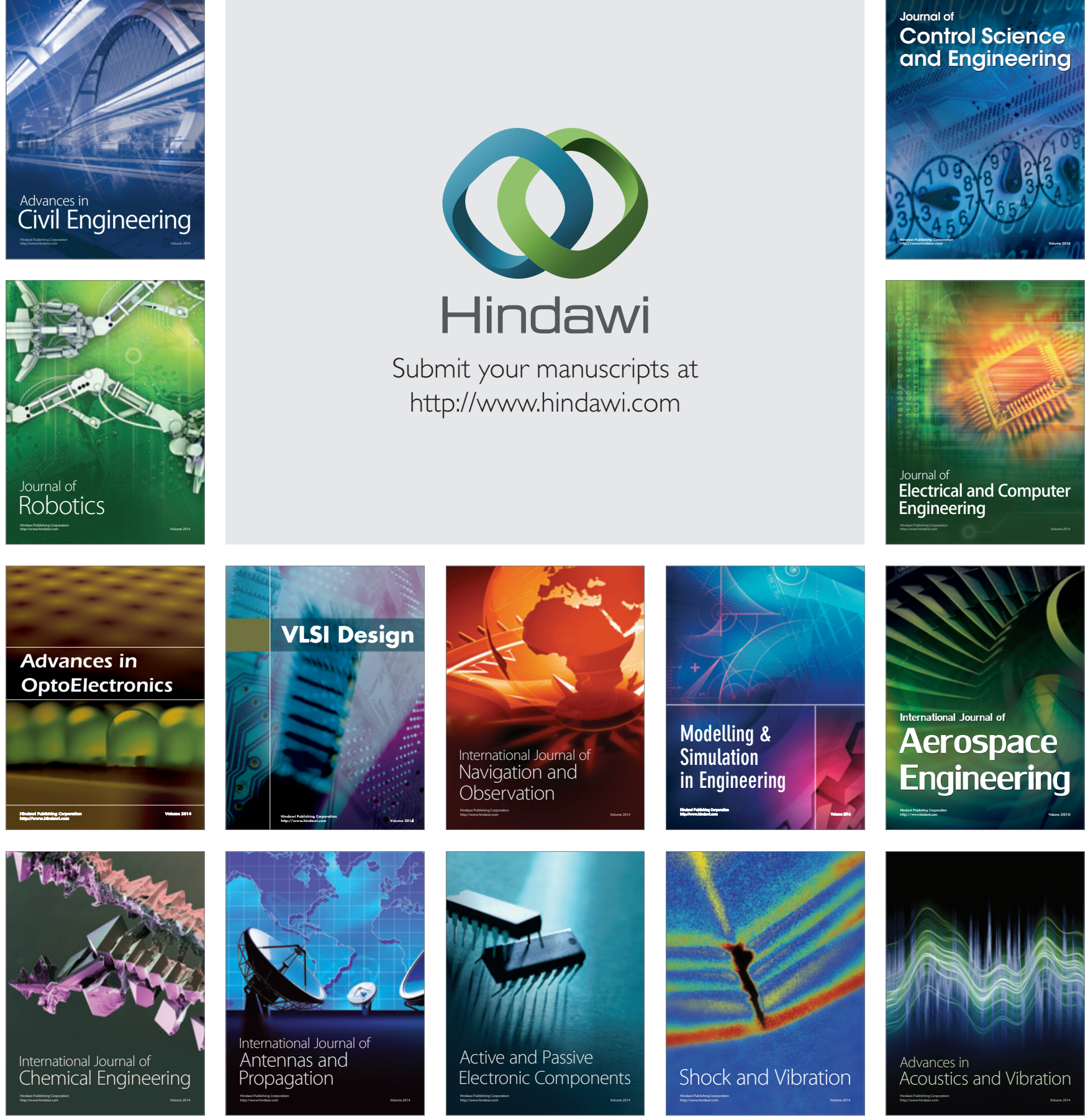